\title{
Les ouvriers en Europe centrale : la dissolution d'une catégorie sociale dans les statistiques
}

Marie Plessz

\section{Notes \& Documents}




\title{
Résumé :
}

L'article vise à établir et à expliquer la très faible visibilité des ouvriers dans les sociétés postcommunistes, en particulier en République tchèque. Pourquoi la crise manifeste que traverse ce groupe social depuis 1989, en contraste si fort avec la situation précédente retient-elle si peu l'attention des sociologues ? Pour répondre à cette question nous évaluons d'abord les ferments d'unité et de division, objectifs et subjectifs, des ouvriers sous le communisme. Puis nous montrons combien les statistiques officielles, surtout en République tchèque, rendent tout décompte précis des ouvriers impossible après 1989. Nous montrons que les publications portant sur les ouvriers sont particulièrement peu nombreuses depuis 1989 alors qu'on peut estimer que les ouvriers représentent toujours entre un quart et un tiers de la main-d'œuvre centre-européenne. Nous proposons plusieurs explications: la situation objective des ouvriers dans des économies en crise et en désindustrialisation; le financement de la recherche sur projets orientés vers des thèmes "à la mode »; le déclin des idéologies et des porte-parole susceptibles de valoriser les identités ouvrières. Nous achevons en relevant les ambiguïtés des discours sociologiques sur les ouvriers.

Pour citer ce document :

Plessz, Marie (2009). «Les ouvriers en Europe centrale : la dissolution d'une catégorie sociale dans les statistiques », Notes \& Documents, 2009-2, Paris, OSC

Pour une version électronique de ce document de travail et des autres numéros des Notes \& Documents de I'OSC, voir le site web de l'OSC : http://osc.sciences-po.fr/publication/pub_n\&d.htm

\begin{abstract}
:
The paper shows that the industrial manual workers have become invisible in post communist societies, especially in the Czech Republic. It tries to explain why a social group which has been in deep crisis since 1989 (contrasting with the communist era) has been forgotten so completely by sociologists. First we analyse what made for unity and diversity among industrial manual workers under communism. Then we show how published statistics make it impossible to count these workers after 1989. The number of publications on these workers in sociological reviews has dramatically decreased. However the industrial manual workers still represent between one quarter and one third of the central European workforce. We then propose some explanation to their invisibility: the objective situation of these workers in times of crisis and deindustrialisation; research funds oriented towards specific topics; the decline of ideologies and of spokesmen who used to strengthen blue-collar workers identities. In the end we underline some ambiguities in the sociological discourses on these workers.
\end{abstract}

Readers wishing to cite this document are asked to use the following form of words:

Plessz, Marie (2009). "Les ouvriers en Europe centrale : la dissolution d'une catégorie sociale dans les statistiques", Notes \& Documents, 2009-2, Paris, OSC, Sciences Po/CNRS.

For an on-line version of this working paper and others in the series, please visit the OSC website at: http://osc.sciences-po.fr/publication/pub_n\&d.htm 
OSC - Notes \& Documents $N^{\circ} 2009-02$

Marie Plessz - Les ouvriers en Europe centrale : la dissolutuion d'une catégorie sociale dans les statistiques

Le débat sur l'(in)existence des classes sociales (et en particulier de la classe ouvrière) a été particulièrement vif en sociologie et est régulièrement réactivé. En France ce débat prend une dimension supplémentaire, d'ordre méthodologique, du fait d'une double tradition. L'intérêt des chercheurs français pour la façon dont les catégories statistiques ou légales façonnent et reflètent les représentations politiques, savantes ou ordinaires du monde social est particulièrement vivace ${ }^{1}$, comme l'attestent les publications reconnues sur les catégories de chômeur (Topalov 1994) ou de cadre (Boltanski 1982 traduit en anglais en 1987) par exemple. D'autre part, et en lien avec ce courant de réflexions, le code des Professions et Catégories socioprofessionnelles (PCS), représente un effort particulièrement original et prolongé pour dépasser la simple nomenclature des professions et construire des regroupements qui aient du sens du point de vue des comportements et des conditions d'existence à la fois sur le marché du travail et dans les autres sphères d'activités (culture, politique, etc.) (Desrosières et Thévenot 1988 ; Chenu 1990). Les PCS ont la particularité d'être régulièrement employées non seulement par la statistique publique qui les a élaborées, mais aussi par les sociologues ainsi que par les acteurs politiques, les partenaires sociaux ou les media. La réflexion sur les classes sociales a été fortement influencée par les catégories socioprofessionnelles, qu'elle utilise très régulièrement (Chauvel 2001), et la moindre utilisation de cette nomenclature dans les publications de l'INSEE récemment attire également l'attention et les réflexions des chercheurs (Pierru et Spire 2008).

L'étude du groupe ouvrier en Europe centrale s'appuie avec fécondité sur cette double tradition française. En mettant en jeu d'autres classifications, souvent purement professionnelles (et non socioprofessionnelles), elle met en relief la spécificité des PCS, et donc du regard que le sociologue français peut jeter sur d'autres traditions statistiques et sociologiques. En montrant des évolutions très marquées par le contexte économique et politique et leur écho dans le monde de la sociologie, elle suggère combien les angles morts de la recherche sociologique sont lourds de sens pour la façon dont une société se représente à elle-même et aux autres. Ce travail est centré sur la République tchèque essentiellement pour des raisons linguistiques, mais nous avons aussi beaucoup mobilisé la Pologne car la situation des ouvriers avant le communisme y contraste avec la Tchécoslovaquie déjà industrielle. Nous essaierons tout d'abord de comprendre si la " classe ouvrière » existait en tant que classe sous le communisme, puis nous montrerons comment la catégorie d'ouvrier devient de plus en plus difficile à saisir dans les statistiques tchèques en particulier, avant de montrer le désintérêt des sociologues centre-européens pour une population ouvrière dont le statut objectif et symbolique s'est fortement détérioré. Précisons cependant que nous ne supposons pas que la représentation des ouvriers en termes de classe serait bonne en soi. II est important de comprendre en quels termes cette catégorie est (ou n'est pas) perçue parce que les représentations sont toujours portées par des acteurs (politiques entre autres) et des institutions et que de

\footnotetext{
${ }^{1}$ Ainsi nos interlocuteurs à l'Institut de philosophie et de sociologie polonais étaient surpris de la précision des questions que nous avons pu poser sur la classification des professions polonaise, dont l'esprit n'est pas très éloigné des PCS (nous y reviendrons). Ces questions nous apparaissaient comme les connaissances nécessaires à acquérir pour pouvoir utiliser correctement cette classification: qui l'a élaborée ? Dans quelle institution (recherche, commission au plan ou statistique publique) ? Quelle en est l'inspiration (marxisme théorique, empirie...) ?
} 
OSC - Notes \& Documents $N^{\circ} 2009-02$

Marie Plessz - Les ouvriers en Europe centrale : la dissolutuion d'une catégorie sociale dans les statistiques

ce fait, elles influencent la définition de la situation ${ }^{2}$, des problèmes et des solutions envisageables. De ce point de vue il n'est certainement pas indifférent que les acteurs aient une vision plutôt gradualiste ou plutôt catégorielle de la structure sociale.

\section{Les ouvriers sous le communisme : fantasme et réalités}

Y avait-il une classe ouvrière sous le communisme ? La réponse à cette question dépend de trois éléments : la définition d'une "classe " que l'on retient bien sûr, mais aussi le pays observé et même la date à laquelle on se penche sur ce pays. La notion de classe implique presque toujours deux ordres de réalités, qui peuvent être plus ou moins hiérarchisés selon les auteurs. Le premier ordre est économique et renvoie à la position spécifique que les ouvriers occupent sur le marché du travail, dans la relation d'emploi et dans les entreprises, souvent aussi aux conditions matérielles d'existence des ouvriers ; le second est symbolique ou politique et renvoie aux identités collectives et au-delà, à la capacité de la classe à se mobiliser en tant qu'acteur collectif. C'est la distinction entre " situation de classe » et " action de classe » chez Weber, ou entre classe pour soi et classe en soi chez Marx. Cette distinction nous sera très utile pour examiner successivement la classe ouvrière fantasmée par le gouvernement communiste, les existences ouvrières sous le communisme et enfin la capacité des ouvriers à se mobiliser en acteurs collectifs. Sur ces deux derniers points les situations polonaise et tchécoslovaque contrastent.

\subsection{Une classe fantasmée par les régimes socialistes}

La « dictature du prolétariat » n'aurait aucune légitimité s'il n'y avait pas de prolétariat : tous les régimes communistes possèdent donc une classe ouvrière, mais c'est une classe ouvrière fantasmée, qui existe non pas tant « pour soi » que " pour eux », pour le pouvoir et par le pouvoir. La propagande officielle et les dispositifs institutionnels s'efforcent de rendre visibles les ouvriers en bons communistes, et les bons communistes en ouvriers. Ainsi les nomenclatures de la statistique publique, en ne distinguant que trois classes (ouvriers, paysans et intelligentsia), fondent en un seul groupe ouvriers et employés (Bafoil 2000). Les quotas à l'université favorisent les personnes qui peuvent se prévaloir d'une appartenance " ouvrière » mais cette appartenance est définie de façon très large : ce peut être un parent ouvrier ou simplement le fait d'avoir travaillé quelques mois comme ouvrier (Kreidl 2005). Ces personnes apparaissent ensuite comme " ouvrières" dans les statistiques. De même les statistiques officielles des partis communistes portent à croire que les définitions ont été élargies puisque la part des ouvriers y aurait augmenté de 40,2 \% à 46,2 \% en Pologne entre 1964 et 1979 (Bafoil 2000), alors même que les directives du parti ne cessent d'inciter les membres à recruter des ouvriers (Gati et Triska 1981). En effet ces derniers ont tendance à sortir du PC ou à en être radiés (en particulier pour alcoolisme et pour non-paiement des cotisations). Mais surtout ils sont très vite submergés par les adhésions de jeunes gens diplômés et/ou

\footnotetext{
${ }^{2}$ Rappelons le théorème de Thomas : « If men define situations as real, they are real in their consequences ».
} 
OSC - Notes \& Documents $N^{\circ} 2009-02$

Marie Plessz - Les ouvriers en Europe centrale : la dissolutuion d'une catégorie sociale dans les statistiques

ambitieux, qui voient dans l'adhésion un moyen voire une condition d'accéder à un poste plus attractif (Možný 1991). Ainsi même parmi ceux qui sont effectivement ouvriers au moment de leur adhésion, beaucoup sont ensuite promus à d'autres types de postes. Enfin bien sûr, toute la rhétorique des PC tient pour évidente l'existence de cette classe ouvrière héroïque et mobilisée pour la construction du socialisme, incarnée sur les affiches par de jeunes gens souriants et enthousiastes, leurs outils à la main. Au final la question de l'existence de la classe ouvrière ne peut pas se poser sous le communisme : ce n'est pas une question, c'est une nécessité idéologique et politique. La légitimité du régime en dépend. Pour se faire une opinion, il faut donc regarder hors des statistiques et des discours officiels, ou du moins dans leurs failles.

\subsection{Réalités: des modes de vie de plus en plus convergents... vers la société de consommation}

Quelle(s) réalité(s) peut-on entrevoir derrière cette mise en scène d'une classe par ses représentants auto désignés ? Commençons par évoquer leurs conditions matérielles d'existence. Un trait commun se dégage des deux pays étudiés, avec des nuances que nous évoquerons par la suite. Les ouvriers communistes forment un groupe social très " jeune " au sens où ses membres sont massivement issus de l'exode rural. Leurs modes de vie s'homogénéisent ensuite mais ils tendent de plus en plus vers un modèle qui n'est pas prolétaire, celui de la société de consommation. En effet, selon le dogme léniniste, l'industrialisation extensive (basée sur un accroissement de la main-d'œuvre) est le principal but à poursuivre en termes de développement économique. Elle permet également d'éloigner le spectre du chômage, un argument de propagande de tout premier plan pour les régimes socialistes ${ }^{3}$. Accroître très brutalement les effectifs ouvriers signifie donc que l'on puise largement dans les réservoirs de main-d'œuvre existants, et en tout premier lieu la main-d'œuvre agricole. Par suite, en Tchécoslovaquie en 1967, 37,5 \% des ouvriers avaient un père paysan et 53,6 \% avaient un père ouvrier. En Pologne ces deux chiffres valaient respectivement $43,2 \%$ et $50,4 \%$ en 1972. D'après une enquête de 1963 un ouvrier polonais sur deux avait vécu à la campagne au moins jusqu'à l'âge de 14 ans (Gati et Triska 1981). Cela nous révèle à quel point la classe ouvrière était jeune et nouvelle en Europe centrale, en particulier en Pologne. En effet vers 1930 il y avait en Pologne $64 \%$ de paysans contre $37 \%$ en Tchécoslovaquie (Gati et Triska 1981). Pour ces personnes qui ont participé à l'exode rural, devenir ouvrier représente une mobilité sociale ascendante, et l'accès à un confort quotidien jusque-là inconnu. Mais bon nombre de ces ouvriers polonais conservent de fortes attaches à la campagne, pour preuve le million d'ouvriers (15\% du total) qui travaillent aussi sur une exploitation agricole en 1974 (Gati et Triska 1981). Ils ont aussi tendance à simplement déplacer leur mode de vie paysan dans leur nouvel espace urbain : par exemple ils vivent essentiellement dans leur cuisine, ou ne vont pas au travail mais à l'Église les jours de fêtes religieuses (Bafoil 2007). Cela signifie aussi que les ouvriers de la "seconde génération" nés en ville, ne ressembleront pas à leurs pères. La situation est probablement légèrement différente en Tchécoslovaquie où l'industrie était plus importante avant-guerre et où l'idéologie communiste avait relativement prospéré sous les hospices de la démocratie, une «classe

\footnotetext{
${ }^{3}$ «En 1979, un sondage réalisé en Pologne montrait qu'avant les salaires, c'est le plein-emploi qui constituait l'élément décisif du soutien accordé au socialisme » (Bafoil 2000).
} 
OSC - Notes \& Documents $N^{\circ} 2009-02$

Marie Plessz - Les ouvriers en Europe centrale : la dissolutuion d'une catégorie sociale dans les statistiques

ouvrière " qualifiée, politisée existait donc déjà en particulier dans les régions les plus industrielles. En outre, le déplacement des frontières polonaises vers l'Ouest a impliqué des mouvements de populations et des changements de nationalité qui troublent encore plus les identités collectives.

D'autre part, la féminisation de la classe ouvrière, bien qu'elle se soit développée dans des secteurs d'activités souvent très spécifiques (textile notamment) et probablement avec une forte ségrégation professionnelle, contribue aussi à diminuer la cohérence des ouvriers comme groupe social. Sur la base de ces origines souvent rurales, l'usine, la ville et les organisations de masse socialisent progressivement ces ouvriers. Mais même au travail, leur communauté de destin est ambiguë. D'une part, la conscience aiguë de la « ligne des cols » qui sépare les ouvriers de " ceux qui sont assis » dans les bureaux (Bafoil 2000), de même que la " conscience critique " développée par les ouvriers sous le socialisme, conscience que le système ne fonctionne pas comme il prétend fonctionner et ne remplit pas ses engagements d'efficacité et d'équité (Burawoy 1988), semblent communes à tous les ouvriers et s'enracine profondément dans la position que les ouvriers occupent dans l'économie planifiée et dans l'entreprise d'Etat, autrement dit dans sa "situation de classe " pour reprendre le terme de Weber. En outre les collectifs de travail étaient souvent très soudés, l'organisation et la coopération étant nécessaires pour remplir les normes de production en situation de pénurie (Burawoy et Lukács 1992). Mais par ailleurs, les divisions sectorielles et en particulier la distinction entre entreprises prioritaires et non prioritaires dessinent des clivages profonds, que ce soit dans les niveaux de salaire ou dans les conditions d'accès aux biens rares comme le logement (Rowell 2006). Ainsi Redor (1988) montre que la différence de salaire entre travailleurs manuels et non manuels est relativement faible, mais que la dispersion des gains est beaucoup plus forte parmi les travailleurs manuels.

Enfin, dans les années 1970-1980, la consommation a pris une valeur nouvelle dans les sociétés communistes, une valeur qui est partagée par tous ses membres, mais qui n'est pas toujours accessible aux ouvriers surtout non qualifiés. Le "socialisme du frigidaire " permet à un grand nombre de Hongrois d'y accéder, mais au prix de journées de travail très longues et souvent contradictoires avec les collectifs de travail. C'est que les Hongrois construisent leur résidence secondaire à la campagne avec des amis le week-end (souvent un simple chalet), travaillent dans l'économie grise après le travail, ou s'impliquent dans les VGMK, des collectifs de travail que l'entreprise paie à la tâche, qui utilisent les équipements de l'usine pour produire hors des horaires de travail. Ces collectifs sont fondés sur l'interconnaissance, le réseau personnel, et les arrangements informels, et ils brouillent le jeu des collectifs de travail « officiels » ('équipe, la brigade de travail, ou l'atelier), créant des lignes de fracture entre ouvriers selon qu'ils sont ou non capables de se procurer de telles opportunités de gains (Burawoy 1988 ; Szalai 2006). De tels dispositifs ont également vu le jour en Pologne dans les années 1980, mais pas en Tchécoslovaquie. Or ces gains supplémentaires sont essentiels car ils permettent la consommation, une aspiration commune aux sociétés communistes et occidentales dans leur ensemble. Ainsi Kolankiewicz (1981) souligne que la crise économique en Pologne à partir de la fin des années 1970 n'a fait qu'aggraver les tensions autour de l'accès aux biens, car les aspirations en matière de consommation matérielle convergent dans l'ensemble de la société mais en situation de pénurie, les privilèges plus ou moins institutionnalisés et rarement légitimes de certaines frange de la main-d'œuvre (en particulier les ingénieurs, disposant de compétences techniques spécialisées) créent des crispations encore plus fortes. Au final dans les années 1950, les 
OSC - Notes \& Documents $N^{\circ} 2009-02$

Marie Plessz - Les ouvriers en Europe centrale : la dissolutuion d'une catégorie sociale dans les statistiques

ouvriers n'ont rien d'une classe car leur socialisation est largement celle de ruraux sans qualification industrielle. La classe ouvrière préexistante en Tchécoslovaquie fait figure d'exception. Au cours du temps, les ouvriers sont à la fois sujets aux effets fédérateurs de leur position spécifique dans l'entreprise industrielle, des politiques centralisées dont ils sont l'objets et de la socialisation produite par les collectifs de travail institués et informels ; et aux effets centrifuges des clivages institués entre différentes entreprises industrielles et de l'aspiration à la consommation qui pousse à chercher de menus profits dans l'économie grise ou en marge du travail à l'usine.

\subsection{Les ouvriers, acteur collectif en puissance?}

Quelle place, dès lors, pour les ouvriers comme acteur collectif en Pologne et en Tchécoslovaquie avant 1989 ? Ici le contraste entre les deux pays est particulièrement instructif. En effet, en Pologne les ouvriers ont été très régulièrement mobilisés collectivement contre le régime communiste, organisant des grèves redoutées par le parti $(1956,1968,1970-1971,1976$, et 1980-1981) et se structurant de plus en plus (le KOR, Comité de défense des ouvriers, naît en 1976 et Solidarité, premier syndicat indépendant dans le bloc communiste est fondé en mai 1981). Solidarité a constitué le point d'orgue de cette mobilisation ouvrière intense. Cependant quelles sont les revendications des ouvriers? elles sont le plus souvent matérielles : protestations contre la hausse des normes de production ou la hausse des prix des biens de consommation de base, contre la modification des grilles de salaires, contre des dysfonctionnements de l'économie qui mettent en péril le versement des primes... Autrement dit, elles émergent des problèmes concrets du quotidien, non d'une remise en cause du système socialiste dans son ensemble et dans ses principes $^{4}$. Au contraire en Tchécoslovaquie, les seules grèves que l'on puisse noter ont lieu en 1953 à Plzeň ainsi qu'à Prague et Ostrava (les trois grandes régions industrielles tchèques) pour protester contre une réforme monétaire qui menace le pouvoir d'achat. En 1968, la population se mobilise essentiellement en réaction à l'entrée des troupes du Pacte de Varsovie dans le pays, pour défendre les réformes du gouvernement lors du Printemps de Prague. Les Tchécoslovaques ne redescendront pas dans la rue avant 1989. Cette apathie signifie-t-elle que les ouvriers tchécoslovaques n'ont pas constitué un acteur collectif tout au long de la période communiste, eux qui avaient pourtant une culture ouvrière plus ancrée et plus répandue que leurs voisins polonais ? II nous semble que cette lecture serait hâtive. Les ouvriers ont plutôt constitué en Tchécoslovaquie un acteur collectif potentiel, une menace de troubles que le régime prenait très au sérieux. Dans les politiques sociales et économiques, il s'agissait justement d'éviter que la masse des travailleurs industriels ne se mobilise. Les ouvriers sont donc une menace de troubles, qui impose de savants dosages entre égalitarisme et stimulation de la productivité, entre la priorité à l'industrie lourde et la nécessité de fournir des biens de consommation ${ }^{5}$. En Tchécoslovaquie on a ainsi renoncé à augmenter la dispersion des rémunérations, dont on espérait pourtant qu'elle accroîtrait la productivité industrielle, par

\footnotetext{
${ }^{4}$ Même s'il ne fait aucun doute que les Polonais étaient spontanément assez hostiles au système communiste, athée et imposé par les Russes.

${ }^{5}$ Voir (Crowley 1997) pour une analyse très fine de la dépendance mutuelle entre la direction des entreprises et les salariés de la production, de son degré variable selon les secteurs et de ses conséquences sur les mobilisations collectives avant et après 1991 en URSS.
} 
OSC - Notes \& Documents $N^{\circ} 2009-02$

Marie Plessz - Les ouvriers en Europe centrale : la dissolutuion d'une catégorie sociale dans les statistiques

peur de la grogne ouvrière (Parkin 1969). En Pologne les ouvriers touchent des primes de charbon et de savon visant à compenser le fait que certaines catégories de travailleurs (les mineurs entre autres) reçoivent en nature ces produits qui sont régulièrement en pénurie (Bafoil 2000). Sous cet angle on comprend mieux les grèves ouvrières parfois violentes qui ont agité la Pologne par exemple à cause d'une hausse du prix de la viande (en décembre 1970), hausse dont l'annulation quelques mois plus tard apporte une popularité inédite au nouveau leader polonais, Gierek. En Pologne, les aspirations à la consommation - qui étaient bien souvent simplement le désir d'en finir avec les pénuries et le rationnement récurrent de tel ou tel produit des plus ordinaires, viande, pain, sucre - ont constamment buté sur la mauvaise santé de l'économie et la priorité à l'industrie lourde. Gierek, arrivé aux commandes suites aux grèves de 1970, s'efforce d'instaurer lui aussi un "socialisme du bigos ", en recourant à l'importation de biens de consommation. Mais l'importation est financée par des emprunts à l'étranger, une stratégie qui n'est pas viable à long terme. Le choc pétrolier de 1973 et ses conséquences sur l'économie mondiale mettent un terme à cette expérience, le rationnement et les pénuries font leur retour et la morosité et la frustration relative reviennent dans les ménages polonais : «on manquait absolument de tout : d'ampoules, de bottes en caoutchouc, de médicaments et de papier hygiénique. [...] En 1978, le taux d'inflation atteignit environ $30 \%$. Les services de sécurité s'en alarmèrent : "le climat social s'aggrave” » (Bafoil 2007). On perçoit ici le lien entre la non-satisfaction des besoins matériels et la frustration et la colère des populations. Celles-ci font peur aux autorités, qui s'efforcent autant que possible "d'acheter de la paix sociale " avec des frigidaires ou des autos en Hongrie, ou simplement avec des produits de première nécessité en Pologne. L'acteur collectif que constituent les ouvriers en Pologne est réalisé en partie à cause de l'incapacité du régime à garantir l'accès à ces biens, encore plus à satisfaire les aspirations à la consommation de masse des Polonais, de plus en plus conscients du décalage entre leur niveau de vie et le niveau de vie occidental. En Tchécoslovaquie, l'acteur collectif est resté une potentialité, une possibilité qu'il faut prendre en compte mais qui n'a pas trouvé de raison de se concrétiser dans des mobilisations collectives. Certainement, il faudrait faire entrer bien d'autres éléments en ligne de compte - ainsi la relative popularité du PC en Tchécoslovaquie en 1945 contraste avec la défiance qu'inspire aux Polonais cette idéologie athée portée par le menaçant voisin russe.

Pour conclure, les régimes communistes ont tous mis en avant, pour asseoir leur légitimité, des classes ouvrières largement fantasmées, qui seraient nombreuses, unies et mobilisées en faveur du régime. Les effectifs ouvriers étaient effectivement importants, mais le noyau, plus ou moins nombreux selon les pays, d'ouvriers de l'entre-deux-guerres est vite submergé par les paysans qui participent à l'exode rural, et dans une moindre mesure par les femmes. Cette population qui partage une commune distance, objective et subjective, avec les " cols blancs ", reste divisée par les lignes de clivages sectoriels ou entre entreprises, et par leur niveau de qualification qui recoupe bien souvent leur origine rurale ou urbaine. Cependant au cours du temps une aspiration vers la consommation de masse se dessine, commune aux ouvriers elle ne leur est pourtant pas spécifique et renvoie plus à un habitus de classe

\footnotetext{
${ }^{6}$ Plat polonais traditionnel à base de chou, contenant aussi des pruneaux et de la viande.
} 
OSC - Notes \& Documents $N^{\circ} 2009-02$

Marie Plessz - Les ouvriers en Europe centrale : la dissolutuion d'une catégorie sociale dans les statistiques

moyenne, assez individualiste, qu'à une " culture ouvrière " telle qu'on l'imagine généralement. Enfin les ouvriers développent une "conscience critique " (Burawoy 1988) qui se nourrit du décalage entre la mise en scène ritualisée du discours officiel sur l'économie et la réalité de son fonctionnement quotidien. Cela fait d'eux un acteur collectif en puissance, dont la capacité à se mobiliser est parfaitement mesurée par les autorités mais généralement motivée par des problèmes d'ordre matériel en particulier autour du niveau de vie. Les régimes politiques s'efforcent donc de satisfaire dans une certaine mesure les aspirations à la consommation des ouvriers communistes, afin de préserver la paix sociale. Les grèves qui n'ont cessé d'agiter la Pologne illustrent ce qui se passe au contraire quand des ouvriers qui disposent de ressources identitaires (le catholicisme, l'identité polonaise rempart contre l'impiété et la barbarie russes), organisationnelles (l'Église puis Solidarité) et intellectuelles (la dissidence polonaise) sont confrontés à une pénurie persistante des biens les plus élémentaires. Pour cette raison, la "classe ouvrière " existait probablement plus en soi en Tchécoslovaquie qu'en Pologne communiste - tout en étant plus mobilisée (autour d'enjeux souvent matériels) en Pologne.

\section{Après 1989 : les ouvriers invisibles ?}

À partir de 1989, l'identité ouvrière ainsi que les conditions objectives d'existence des ouvriers sont radicalement modifiées. L'idéologie qui en avait fait des "héros au pouvoir " (Bafoil 2000) est profondément dévalorisée, les restructurations et fermetures d'usines signifient pour beaucoup au mieux la préretraite, au pire le chômage. Or quelle peut être l'identité d'un ouvrier chômeur ? La faiblesse des mobilisations et des affiliations syndicales et politiques des chômeurs en dit long sur l'absence de statut que signifie le chômage. Au cours de notre travail de recherche, notre première intention était de décrire les conditions d'existence actuelles des ouvriers, un angle mort dans la littérature sur la transformation postcommuniste. Confrontée à l'impossibilité de construire de telles données en particulier en République tchèque, nous suggérons ici que le premier fait concernant les ouvriers après 1989, est leur invisibilité. Cette invisibilité est d'abord statistique, elle est plus ou moins marquée selon les pays, du fait de choix différents en matière de nomenclatures. Mais elle renvoie aussi à une certaine cécité des chercheurs, dont il faudra chercher les limites et les explications. Nous passerons ensuite en revue ce que les rares auteurs qui ont étudié la question nous disent sur les ouvriers après 1989.

\subsection{Le flou statistique autour des ouvriers aujourd'hui}

Szalai (2006) estime que les ouvriers « représentent $40 \%$ de la société hongroise », mais ne précise pas comment elle obtient ce chiffre, dans quelle nomenclature et sur quelles données. Que l'on puisse lancer de telles affirmations sans même se référer à des données de la statistique publique en dit long sur le flou qui règne sur la place des ouvriers dans les sociétés postcommunistes, ne serait-ce que du point de vue quantitatif. Le cas tchécoslovaque est le plus extrême. Le choix du code des professions ISCO comme seule nomenclature des professions, et l'anonymisation des données accessibles au chercheur font qu'il est impossible de compter les ouvriers en République tchèque depuis 1989. Par là nous entendons qu'il est impossible d'identifier les ouvriers (même regroupés avec les ouvriers agricoles comme c'est le cas dans 
OSC - Notes \& Documents $N^{\circ} 2009-02$

Marie Plessz - Les ouvriers en Europe centrale : la dissolutuion d'une catégorie sociale dans les statistiques

les PCS françaises au niveau agrégé) dans des données qui couvrent un échantillon suffisamment vaste pour permettre des estimations avec de faibles marges d'erreurs. L'enquête Social Stratification in Eastern Europe, permet, en effet, d'identifier les ouvriers (routine manual workers) au sens de la classification $E_{G P}{ }^{7}$, mais les effectifs de quelques milliers de personnes contenus dans l'échantillon nous paraissent insuffisants. Nous voudrions donc pouvoir compter les ouvriers de l'industrie dans une enquête aux effectifs importants, comme les Labour Force Surveys les recensements ou les Microcensus. Mais, sauf à être membre du Bureau tchèque de la Statistique, cela est impossible.

En effet, les données des LFS publiées par le CZSO comme par Eurostat sont anonymisées de façon très restrictive. Le code ISCO est tronqué après le deuxième chiffre. Or le code ISCO à deux chiffres ne suffit pas à identifier les ouvriers. En effet ceux-ci sont répartis dans trois grands groupes. Le groupe 7 (artisans et ouvriers des métiers de type artisanal), le groupe 8 (conducteurs d'installations et de machines et ouvriers de l'assemblage) qui est le seul à contenir uniquement des ouvriers, et le groupe 9 (ouvriers et employés non qualifiés). Dans le groupe 9 on trouve :

- $91 \quad$ Employés non qualifiés des services et de la vente ;

- 92 Manœuvres de l'agriculture, de la pêche et assimilés ;

- 93 Manœuvres des mines, du bâtiment et des travaux publics, des industries manufacturières et des transports.

Dans le groupe 7 par contre, il est quasiment impossible de déterminer qui est ouvrier à proprement parler et qui ne l'est pas. En effet la classification ISCO est une classification des professions uniquement. Les groupes sont basés sur le niveau de qualification normalement requis pour réaliser les tâches impliquées par un métier. Le statut d'emploi (indépendant ou salarié) n'entre pas en jeu. Or dans le groupe 7 on trouve des professions très diverses. Nous présentons ici les regroupements de métiers du groupe 7 avec un niveau de détail variable selon les sous-groupes, de façon à en montrer l'hétérogénéité :

71 Artisans et ouvriers des métiers de l'extraction et du bâtiment

72 Artisans et ouvriers des métiers de la métallurgie, de la construction mécanique et assimilés

-721 Mouleurs de fonderie, soudeurs, tôliers-chaudronniers, monteurs de charpentes métalliques et assimilés

7211 Mouleurs et noyauteurs de fonderie

7212 Soudeurs et oxycoupeurs

7213 Tôliers-chaudronniers

7214 Charpentiers métalliers et monteurs de charpentes métalliques

7215 Gréeurs et épisseurs de câbles

7216 Scaphandriers et hommes-grenouilles

- 722 Forgerons, outilleurs et assimilés

73 Artisans et ouvriers de la mécanique de précision, des métiers d'art, de l'imprimerie et assimilés

- 731 Mécaniciens de précision sur métaux et matériaux similaires

7311 Mécaniciens-réparateurs d'instruments de précision

7312 Facteurs et accordeurs d'instruments de musique

7313 Joailliers et orfèvres

- 732 Potiers, souffleurs de verre et assimilés

\footnotetext{
${ }^{7}$ La classification élaborée par Erickson, Goldthorpe et Portocarrero fait figure de référence hors de France. Elle est probablement la représentation d'une structure de classe la plus proche des PCS même si son esprit est légèrement différent puisqu'elle se bâtit sur la notion de " relation d'emploi »(Erikson, Goldthorpe et Portocarero 1979).
} 
OSC - Notes \& Documents $N^{\circ} 2009-02$

Marie Plessz - Les ouvriers en Europe centrale : la dissolutuion d'une catégorie sociale dans les statistiques

- 733 Ouvriers des métiers d'artisanat sur bois, sur textile, sur cuir et sur des matériaux similaires

- 734 Artisans et ouvriers de l'imprimerie et assimilés

74 Autres artisans et ouvriers des métiers de type artisanal

- 741 Artisans et ouvriers de l'alimentation et assimilés

Ainsi au sein du sous-groupe 71 on retrouve aussi bien les mineurs que les ouvriers du bâtiment, deux types d'ouvriers très différents. Le sous-groupe 72 et le sous-groupe 73 en revanche, mêlent des professions susceptibles d'employer des ouvriers de métier, qualifiés, ayant une forte tradition d'identité ouvrière, comme le mouleur ou le réparateur d'instruments de précision, et des professions qui n'ont rien d'ouvrières, comme l'homme-grenouille ou l'accordeur d'instruments de musique.

II est donc très hasardeux d'utiliser cette seule variable dans son codage à deux chiffres pour isoler les ouvriers dans les LFS. On pourrait s'en approcher en utilisant le statut d'emploi (et exclure les artisans indépendants) ainsi que la branche d'activité (et ne conserver que les personnes travaillant dans une entreprise industrielle). Mais on sait que le codage de la branche s'appuie sur l'activité principale de l'entreprise, ce qui peut induire en erreur. Aussi peut-on affirmer qu'aucune publication du CZSO (pas plus que celles d'Eurostat d'ailleurs) ne permet de dire combien il y a actuellement d'ouvriers en République tchèque. L'accès aux données anonymisées des LFS par le biais du réseau Equalsoc n'a que partiellement permis de contourner cette difficulté, mais la majorité des chercheurs, se serait-elle intéressée à la question des ouvriers n'aurait pu en donner une évaluation chiffrée ${ }^{8}$.

En outre les Labour Force Surveys regroupées et anonymisées par Eurostat ne contiennent que peu d'informations susceptibles de nous renseigner sur les conditions de vie et de travail des ouvriers (ainsi pour les pays qui ont relevé les salaires, cette variable n'a pas été conservée). Tout d'abord, pour isoler une catégorie d'ouvriers, nous avons mobilisé deux variables. Le code ISCO a permis d'isoler les professions que l'on retrouve dans le groupe socioprofessionnel des ouvriers en France (en regroupant les groupes 7 et 8 ainsi que le sous-groupe 93). Le code NACE nous a permis d'ajouter le critère de la branche d'activité (nous avons conservé les mines, les transports, l'industrie, la construction et l'énergie). Les ouvriers ainsi définis représentent, de 1997 à $2004^{\circ}$, une part constante et non négligeable de la population en emploi centre-européenne : $32 \%$ en République tchèque, $30 \%$ en Hongrie, $24 \%$ en Pologne. En France avec cette définition on trouve $20 \%$ d'ouvriers jusqu'en 2002 puis $18 \%$ (du fait d'un changement dans la grille de passage des PCS au code ISCO), ce qui sous-évalue d'environ 4 points la population ouvrière telle qu'on la trouve dans le groupe 6 des PCS avec les mêmes données. Parmi ces ouvriers, il reste de $8 \%$ (Pologne) à $12 \%$ (République tchèque) d'indépendants ou chefs d'entreprise. Parmi les ouvriers centre-européens on compte environ un quart de femmes, un peu moins en Pologne $(20 \%)$ sans doute du fait de l'importance persistante du secteur minier. Cette proportion est légèrement plus élevée

\footnotetext{
${ }^{8}$ Cela est d'autant plus vrai qu'aucune institution tchèque n'est membre du réseau Equalsoc. Or pour accéder aux données, il faut être membre d'une institution qui soit membre du réseau (c'est le cas du CNRS en France) ou collaborer sur un projet du réseau.

${ }^{9}$ Nous avons travaillé sur le second trimestre de chaque année civile. II n'y a pas d'information sur le code NACE en Pologne avant 2000 ni en Hongrie en 1998 et 1999 donc nous n'avons pas d'information sur les ouvriers à ces dates.
} 
OSC - Notes \& Documents $N^{\circ} 2009-02$

Marie Plessz - Les ouvriers en Europe centrale : la dissolutuion d'une catégorie sociale dans les statistiques

qu'en France, et peut s'expliquer de deux façons : une féminisation des métiers ouvriers qui signerait le déclin de ces professions, ou une spécificité liée au développement de la structure de l'emploi en Europe centrale $^{10}$. En effet alors qu'en France les femmes accèdent à l'emploi à un moment où les emplois de services se développent rapidement, en Europe centrale ce sont les emplois industriels qui sont alors en expansion très forte. Cela est compatible avec la très forte segmentation des emplois industriels en secteurs masculins et féminins, mais les données sur la branche d'activité ne sont pas assez précises dans les LFS pour nous permettre d'approfondir la question. Au final dans les années 2000 on compte parmi les hommes en emploi $43 \%$ d'ouvriers en République tchèque, $41 \%$ en Hongrie et aux alentours de $34 \%$ en Pologne alors que parmi les femmes on dénombre respectivement $17 \%, 17 \%$ et $10 \%$ d'ouvrières. Pour le reste, les données des LFS ne révèlent rien de bouleversant : les ouvriers sont plus souvent en CDI que les autres personnes en emploi et leur temps de travail hebdomadaire, légèrement supérieur à la moyenne nationale, suggère qu'il y a moins de temps partiels parmi les ouvriers. Leur répartition par âge est très proche de celle de l'ensemble des actifs, peut-être très légèrement décalée vers les groupes plus jeunes, ce qui n'est pas surprenant si l'on se souvient que les professions ouvrières sont réputées être des professions « jeunes » au sens ce sont des professions peu qualifiées, d'entrée sur le marché du travail, ouvrant éventuellement la porte à d'autres professions plus qualifiées (Blau et Duncan 1967).

Par ailleurs on trouve dans les Microcensus tchèques une variable qui code le « groupe social de la personne à la tête du ménage » dont les modalités sont : ouvrier, salarié ${ }^{11}$, indépendant hors agriculture, indépendant dans l'agriculture, retraité dans un ménage dont au moins un membre travaille, retraité dans un ménage dont aucun membre ne travaille. Les chefs de ménages qui sont ouvriers représentent $31,5 \%$ des chefs de ménages en 1996 (24\% en 2002), un chiffre qui ne dit pas grand chose sur la taille du groupe ouvrier, encore moins sur ses caractéristiques sociales puisque les Microcensus sont centrés sur la structure des revenus.

Ainsi la statistique publique tchécoslovaque présente un angle mort autour des ouvriers qu'aucune publication ne permet de compter. Les données détaillées accessibles à un petit nombre de chercheurs sont si fortement anonymisées qu'elles obligent à une gymnastique complexe de recodage dont le résultat est incertain (quand à sa fécondité, étant donné la quantité d'information qui a été retirée du fichier, on peut en douter). Le groupe ouvrier semble ainsi s'être dissous dans la statistique publique. Le choix de la classification ISCO comme seule classification des professions en République tchèque, et comme principal outil de codage dans les données de la statistique publique et dans les enquêtes menées par les instituts de recherche, contribue à opacifier les limites du groupe ouvrier. L'impossibilité de passer avec rigueur du code ISCO à la classification EGP sans mobiliser une autre variable rendant compte du statut d'emploi montre bien que le code ISCO est probablement inapproprié pour un travail sociologique, car les grands groupes qu'il dessine laissent dans l'ombre des variables essentielles de structuration des professions en

\footnotetext{
${ }^{10}$ Nous avions effectivement souligné dans notre thèse que les professions typiquement masculines sont plus féminisées dans les économies communistes que dans les économies capitalistes.

${ }^{11}$ Le mot tchèque zaměstnanec signifie employé mais s'oppose aussi à employeur, zaměstnavatel donc salarié.
} 
OSC - Notes \& Documents $N^{\circ} 2009-02$

Marie Plessz - Les ouvriers en Europe centrale : la dissolutuion d'une catégorie sociale dans les statistiques

groupes socioprofessionnels (le statut de salarié étant l'exemple le plus frappant). Le fait qu'en République tchèque il n'existe pas d'autre classification, à l'opposé de la Hongrie qui possède FEOR (qui ressemble de plus en plus à ISCO) ou de la Pologne (avec la SKZ, Classification sociale des professions, élaborée en 1974 par Słomczyński entre autres sur des bases qui se disent marxistes mais en fait très proches de Weber, retravaillée depuis) révèle selon nous la position de faiblesse des sociologues vis-à-vis du Bureau de la statistique, faiblesse qui découle de son existence chaotique sous le communisme. Mais elle révèle aussi le peu d'intérêt des sociologues pour un groupe social en situation de fort déclassement collectif.

\subsection{Cécité des chercheurs?}

Nous avons tenté d'évaluer l'intérêt que les chercheurs en sociologie pouvaient porter aux ouvriers dans leurs publications, avant et après 1989. Pour cela nous avons construit un indicateur extrêmement simple. Nous avons fait une recherche sur les titres des articles publiés dans les principales revues de sociologie polonaises et tchèque. Les revues retenues ont été : Studia Socjologiczne pour la Pologne, publiée par I'Institut de philosophie et de sociologie, et la Sociologický časopis/Czech Sociological Review en République tchèque, publiée par l'Institut de sociologie.

Nous avons cherché, dans les titres d'articles publiés dans ces revues et archivés par Sociological Abstracts $^{12}$, les occurrences du terme " ouvrier » comme adjectif ou comme substantif, en tchèque pour la revue tchèque, en polonais pour les revues polonaises. Nous avons réalisé la même recherche sur les résumés des articles pour des résultats très proches, les résultats présentés concernent donc uniquement les titres. Cet indicateur rudimentaire est forcément imparfait, d'une part parce que les sociologues publient aussi des livres, d'autre part parce que les revues que nous avons sélectionnées ne couvrent pas tout le champ de la sociologie, en particulier la sociologie qualitative est peut-être sous-représentée. Mais notre mesure bibliographique n'a pas pour but de rendre compte précisément de toute la recherche qui a pu se faire sur les ouvriers, plutôt de saisir des évolutions. D'autre part, nous avons sélectionné des revues dont les archives sont accessibles en ligne afin de simplifier la tâche, et pour compenser le possible biais en faveur des méthodes quantitatives et des thèmes liés à la structure sociale, très présent en particulier dans la Czech Sociological Review, nous avons également passé en revue les tables des matières de la revue Biograf, revue fondée en 1994 par Konopásek (2000) et qui se décrit comme un « Journal not only for biographical and reflexive sociology ». Nous n'avons trouvé aucune occurrence du mot " ouvrier », comme substantif ou comme adjectif dans les titres d'articles publiés par cette revue.

Les résultats de cette recherche sont présentés dans le Tableau 1.

\footnotetext{
${ }^{12}$ http://www.csa.com/factsheets/socioabs-set-c.php.
} 
OSC - Notes \& Documents $N^{\circ} 2009-02$

Marie Plessz - Les ouvriers en Europe centrale : la dissolutuion d'une catégorie sociale dans les statistiques

Tableau 1 : Titres d'articles contenant « ouvrier » dans deux revues polonaise et tchèque

\begin{tabular}{|c|c|c|c|c|c|}
\hline & \multirow[b]{2}{*}{$\begin{array}{l}\text { Première année } \\
\text { référencée }\end{array}$} & \multirow[b]{2}{*}{$\begin{array}{c}\text { Titre le plus } \\
\text { ancien contenant } \\
\text { « ouvrier » }\end{array}$} & \multicolumn{3}{|c|}{ Nombre d'articles } \\
\hline & & & Avant 1989 & Après 1989 & Total \\
\hline $\begin{array}{l}\text { Czech Sociological } \\
\text { Review }\end{array}$ & 1965 & 1983 & 23 & 2 & 25 \\
\hline $\begin{array}{l}\text { Studia } \\
\text { socjologiczne }\end{array}$ & 1961 & 1963 & 21 & 1 & 22 \\
\hline
\end{tabular}

Source : Sociological abstracts. Nous avons tronqué le mot tchèque et le mot polonais signifiant ouvrier de façon à obtenir tous les résultats contenant « ouvrier » comme adjectif, comme substantif, et sous toutes les déclinaisons, en recherchant respectivement dělní $i^{*}$ et robotn* (l'étoile représente n'importe quel caractère ou chaîne de caractère dans la recherche).

On constate la quasi-disparition des ouvriers du paysage académique tel que les deux revues sélectionnées le donnent à voir ${ }^{13}$. Les ouvriers sont-ils tombés dans l'oubli de la part des chercheurs ? Si oui, pourquoi ? Les ouvriers sont une catégorie sociale relativement facile à définir (même si les entraves statistiques à leur simple comptage se sont multipliées comme on l'a vu) et nombreuse (donc présents dans toutes les enquêtes statistiques, et non négligeable dans la structure sociale). L'intérêt des sociologues est bien souvent attisé par les situations de crise, de perte de statut, de déclassement, par l'expérience des dominés: de ce point de vue les ouvriers semblent être un objet de choix. Ainsi les études ne manquent pas en France sur les ouvriers à l'épreuve de la crise de l'emploi industriel (Beaud et Pialoux 1999 par exemple). De même les Roms font couler beaucoup d'encre même en République tchèque où ils ne représentent que $3 \%$ de la population. Ils sont l'objet d'études régulières de la part de la communauté scientifique ainsi que de rapports d'experts pour des organisations supranationales ou non gouvernementales. Comment interpréter alors le désintérêt voire le refus d'intérêt de la part des chercheurs d'Europe centrale à l'égard des ouvriers ? Plusieurs dimensions peuvent être évoquées. La première, la plus évidente, est sans doute le rejet des catégories et des thèmes marxistes (Sztompka 2002), mêlé sans doute au soulagement de pouvoir choisir son objet d'études et donc de laisser derrière soi un objet que le régime socialiste avait sans doute beaucoup encouragé. Mais au-delà de ce rejet du passé, le regard des chercheurs se porte spontanément vers des groupes sociaux nouveaux, qui émergent de la transformation, qu'ils soient les signes de son ambivalence ou la marque de sa réussite. On s'est donc bien plus intéressé aux entrepreneurs ou aux chômeurs (dont beaucoup sont d'anciens ouvriers) qu'aux ouvriers eux-mêmes. Enfin, le financement de la recherche se fait de plus en plus en Europe centrale sous la forme de financement sur projets, par appels d'offres d'un organisme national ou supra national (I'Union européenne entre autres). Cela est vrai aussi en France, mais en Europe centrale les salaires de base des chercheurs

\footnotetext{
${ }^{13}$ On peut s'étonner que les ouvriers apparaissent subitement dans la Revue tchèque de sociologie au début des années 1980. Les articles des numéros des années 1965-1966 font presque tous référence au monde du travail industriel et semblent orientés vers la résolution des problèmes visibles dans le fonctionnement de l'économie socialiste. Cette perspective est manifestement la réponse à une demande des autorités inquiètes de la crise économique du début des années 1960.
} 
OSC - Notes \& Documents $N^{\circ} 2009-02$

Marie Plessz - Les ouvriers en Europe centrale : la dissolutuion d'une catégorie sociale dans les statistiques

étant encore plus modestes, obtenir de tels crédits de recherche est essentiel. Or ces financements sont orientés vers des thématiques particulières. L'Union européenne par exemple finance beaucoup de projets sur la société civile ou sur l'impact des politiques sociales (inégalités de genre et d'origine ethnique, minorités, pauvreté, chômage...). Si les relations professionnelles sont un sujet porteur, les ouvriers eux ne le sont pas. La plupart des publications sur les ouvriers sont donc l'œuvre de chercheurs souvent étrangers (Burawoy, Verdery, Bafoil), souvent de gauche ou du moins critiques avec une inspiration marxiste (Burawoy ${ }^{14}$, Szalai).

Enfin un dernier niveau d'explication peut être proposé : il renvoie à la faiblesse des porte-parole des ouvriers après 1989. Proposer une lecture de la structure sociale qui fasse une place aux ouvriers en tant qu'ouvriers (et non en tant que population peu qualifiée, ou particulièrement vulnérable au chômage et à la pauvreté, ou comme " perdants de la transition ») n'a en effet rien d'évident. Boltanski (2008) suggère ainsi qu'en France,

«La division de la société en classes sociales, le fait que ce principe de classification et ce facteur d'inégalités l'emportaient sur les autres, se présentait aussi pour nous [sociologues proches de Bourdieu] comme une cause, la cause des classes sociales, dont il fallait assurer la défense contre ceux qui en niaient l'importance ou en prédisaient la fin comme pour mieux les effacer de la perception collective, les rendre invisibles ».

Cette cause de la représentation en classes sociales doit donc être défendue, non seulement par les sociologues, mais aussi par des porte-paroles de ces classes qui les fassent exister dans les discours médiatiques et politiques. Ainsi selon Boltanski la représentation en termes de classes pâtit du «nouvel esprit » du capitalisme qui émerge en France dans les années 1980-1990 et qui "n'a pas eu pour conséquence une diminution du poids relatif de l'État mais la mise en place d'une nouvelle formule d'État dont la notion même de classe sociale était absente » (p. 132). La désyndicalisation et l'affaiblissement du PC ont également joué un rôle. Or qu'observe-t-on en Europe centrale ? L'affaiblissement des syndicats est patent au point qu'on a parlé de labour quiescence (Crowley et Ost 2001). En effet les syndicats perdent leur légitimité du fait de leur positionnement idéologique contradictoire (ils ne peuvent se réclamer du marxisme pour s'opposer au patronat mais doivent se démarquer de leur ancien rôle de "courroie de transmission »), de leurs marges de manœuvre très étroites (ils se contentent bien souvent d'essayer d'endiguer les licenciements, ce qui ne saurait satisfaire leur base), et des difficultés qu'il y a à négocier avec un patronat éclaté, changeant et très méfiant. En outre les restructurations, les créations d'entreprises et en particulier de PME ont fortement réduit les effectifs des syndicats. Même Solidarité en Pologne n'a pas résisté aux premières années de démocratie : gouverner et instaurer la " thérapie de choc » lui a attiré l'antipathie de beaucoup d'ouvriers puis le syndicat s'est divisé en courants et partis hétéroclites. Quant aux mobilisations collectives, il y en a eu peu en Pologne, en Tchécoslovaquie et en Hongrie - à la différence de la Roumanie par exemple dont les mineurs ont régulièrement fait parler d'eux. Enfin si le « nouvel esprit

\footnotetext{
${ }^{14}$ Burawoy qui se décrit comme « unrepentant marxist » écrit ainsi : "The working class may betray the intellectuals' hopes and aspirations for emancipation, but this is no reason for intellectuals as a group to then return the favor by betraying the working class, that is by abandoning the working class for some other class upon which it can ride to power. To turn from the working class to the study of elites and policies because they appear to be forging history is to abandon the critical perspective of the subaltern » (Burawoy 2002).
} 
OSC - Notes \& Documents $N^{\circ} 2009-02$

Marie Plessz - Les ouvriers en Europe centrale : la dissolutuion d'une catégorie sociale dans les statistiques

du capitalisme » français est aveugle aux classes, que dire de "l'esprit du nouveau capitalisme " centreeuropéen, qui se construit contre le régime communiste arc-bouté, justement à une vision du social et du politique en termes de classes? Ainsi c'est une multitude de facteurs qui se renforcent mutuellement qui concourent à rendre les ouvriers invisibles dans les sociétés postcommunistes. Ils ont peu d'intérêt pour les chercheurs, ils sont absents des statistiques et ils manquent de porte-parole dans l'espace public, un espace public où les représentations pertinentes de la société ne sauraient emprunter des catégories chères au régime socialiste. Cependant, comme nous l'avons dit, quelques chercheurs, étrangers à l'Europe centrale pour la plupart, on écrit sur les ouvriers.

\subsection{L'ambiguïté des discours sociologiques sur les ouvriers}

Que nous apprennent les travaux consacrés aux ouvriers ? II faut y relever au moins deux ambiguïtés. Tout d'abord ces chercheurs relèvent l'appauvrissement des ouvriers, leur perte de pouvoir et de statut sur le lieu de travail et dans la société et au final leur relative apathie politique. Mais cette situation est-elle spécifique aux ouvriers ? Ainsi Szalai met en relief les nouveaux clivages au sein de la population ouvrière, clivages selon des critères sectoriels propres à une économie capitaliste tournée vers les exportations, mais aussi clivages générationnels :

« D'une manière générale, on peut affirmer qu'un nouveau processus de différenciation majeure est en cours chez les ouvriers. Le principe de ce processus est le suivant, les ouvriers de l'industrie lourde se retrouvent au bas de l'échelle sociale et sont définitivement déclassés, sombrant dans le chômage de longue durée et/ou étant contraints de vive de travaux mal payés ou occasionnels. Ceux qui travaillent dans les branches compétitives de l'industrie (compétitives surtout sur les marchés occidentaux) et dans le secteur des services qui est en pleine croissance, même s'ils ne sont pas capables d'augmenter leurs revenus de façon significative et d'améliorer leur statut social, sont au moins en mesure de le maintenir. Ce second groupe évolue seulement pour une petite partie par le transfert d'ouvriers à ces nouveaux secteurs et plus encore par l'entrée de nouvelles couches d'ouvriers plus jeunes, dont des personnes diplômées ou hautement qualifiées contraintes d'accepter la situation de sous-emploi [...]. Alors que les ouvriers âgés affichent leur amertume, les jeunes, eux, sont apathiques. Ils n'expriment pas d'opinions, même concernant leurs conditions de vie actuelles. Ils refusent une identité ouvrière et se considèrent comme des "employés"15. Parmi les plus âgés, plusieurs se disent membres d'un syndicat, "par habitude", mais les jeunes n'y adhèrent pas » (Szalai 2006).

Les ouvriers interrogés par Bafoil sont inquiets pour leur avenir car le licenciement peut arriver n'importe quand et "la vie est très, très dure, très dure " (Bafoil 2000). Burawoy et Verdery (1999) montrent comment les rythmes de travail se sont accélérés. Mais le travail de Portet suggère que pour la plupart des Polonais, la relation d'emploi est devenue plus prenante et en même temps plus précaire. D'autre part la paralysie des syndicats, si elle nuit beaucoup aux ouvriers, est également très pénalisante pour les employés des grandes entreprises de services (supermarchés, sociétés de nettoyage...). II suggère enfin que la menace du chômage, dans un pays comme la Pologne, pèse la grande majorité des salariés (Portet 2006). La situation des ouvriers est-elle vraiment un cas à part ? Le sort des ouvriers est-il vraiment pire que celui des employés de type industriel que sont les caissières et les agents de nettoyage étudiés par Portet? Et donc, la distinction des ouvriers parmi la masse des salariés d'exécution peu

\footnotetext{
${ }^{15}$ Monchatre (2004) note l'abandon du terme d'ouvrier pour celui d'opérateur en France.
} 
OSC - Notes \& Documents $\mathrm{N}^{\circ} 2009-02$

Marie Plessz - Les ouvriers en Europe centrale : la dissolutuion d'une catégorie sociale dans les statistiques

qualifiés a-t-elle encore un sens ? Nous nous contenterons de donner un chiffre, tout rudimentaire qu'il soit. Dans les données des LFS de 2003 nous avons rapporté la part de chômeurs ayant auparavant occupé un emploi industriel à la part d'actifs actuellement occupés dans l'industrie. Ce chiffre, qui vaut 1,22 en République tchèque (2004), 1,25 en Hongrie et 1,32 en Pologne (en regroupant les secteurs de l'industrie, des mines et de la construction) témoigne de la sur-représentation d'un passé industriel parmi les chômeurs. Autrement dit il semble bien que l'industrie ait plus souvent licencié et que ces chômeurs aient moins que les autres trouvé un nouvel emploi. La situation de fragilité des ouvriers n'est donc peut-être pas spécifique à ces derniers, mais elle semble plus marquée pour eux que pour les autres actifs.

D'autre part, il se dégage de tous ces travaux l'impression que "c'était mieux avant ». Mais les formes de cette nostalgie sont très proches non seulement de ce qu'on peut lire à propos des ouvrier français (Beaud et Pialoux 1999), mais aussi, sous la plume d'un Burawoy (1988) de la part d'ouvriers hongrois dans les années 1980. La perte de pouvoir des syndicats et partant la difficulté à se faire représenter est partout mentionnée (Kramer 1995). La place prise par des machines plus riches en technologies, qui rendent les vieux savoir-faire inutiles et justifient une " logique des compétences » contre l'ancienne logique de l'ancienneté est dénoncée par les ouvriers interrogés (Bafoil 2000). La position de force des patrons pour imposer des licenciements ou repousser des hausses de salaire paraît insurmontable. Pour Szalai (2006) l'exploitation est maintenant « totale » car la vulnérabilité, la pauvreté et l'atomisation des ouvriers a augmenté. Mais ces éléments ne sauraient surprendre le lecteur français, à qui ils sont déjà familiers. Comment expliquer le parallélisme des situations ? Bafoil (2000) cherche des explications à la nostalgie ouvrière du communisme (nostalgie bien compartimentée : on ne regrette pas le communisme en bloc mais certains aspects). II évoque pour cela l'habitus forgé sous le communisme et les logiques d'action des ouvriers, qui font qu'ils ne perçoivent pas la situation comme la direction des usines voudrait qu'ils le fassent. Szalai (2006) avance l'idée que le rapport de classe qui s'est instauré en Hongrie après 1989 n'est pas né directement du contexte hongrois, il est aussi largement importé par les multinationales dans un environnement mondialisé : selon elle, devant l'épuisement des sources de profit, les "élites économiques » internationales ont cherché à briser les acquis sociaux du capitalisme ainsi que les " expérimentations collectives » telles que le socialisme et c'est dans ce monde que la Hongrie fait sa transition au capitalisme.

Plus surprenante peut-être est la proximité des discours tenus après 1989 et des propos recueillis dans une aciérie hongroise par Burawoy dans les années 1980 :

«Pendant quinze minutes nous pouvons nous réfugier dans notre abri cubique [...]. J'y entends les évocations interminables du passé, quand les sidérurgistes étaient vraiment des sidérurgistes. Gyuri, notre chef d'équipe, qui a gagné d'innombrables médailles et titres honorifiques, raconte le bon vieux temps des fours Martin quand il n'y avait pas d'ordinateur pour fixer la quantité de ferraille, de saumon de fonte [...]. "Nous devions juger par nous-mêmes. L'expérience comptait vraiment. Maintenant n'importe quel paysan sans qualification venant d'une coopérative peut être fondeur". Joszi, 45 ans, le plus vieux de la brigade, dont le père avait été une grande figure des laminoirs, dit qu'il ne souhaiterait jamais que son fils suive ses traces. [...] Mais n'est-ce pas mieux aujourd'hui plus sûr, moins chaud? Csaba, qui est de la jeune génération, est le premier à en convenir. Les autres montrent plus d'ambivalence à l'égard de la perte des anciennes qualifications, même lorsqu'elle s'accompagne d'une amélioration des conditions de travail. [...] ils [gardent] la nostalgie d'un rythme de travail qu'ils pouvaient contrôler. La vie était plus dure mais plus humaine » (Burawoy 1988). 
OSC - Notes \& Documents $N^{\circ} 2009-02$

Marie Plessz - Les ouvriers en Europe centrale : la dissolutuion d'une catégorie sociale dans les statistiques

Qui pourrait croire que ces propos ont été recueillis avant 1989 ? Les ouvriers ne savent-ils donc que se plaindre ? Le changement est-il toujours un défi trop insurmontable pour eux ? Certes non. Dunn (1999) en donne un exemple dans un entretien avec une syndicaliste dans une usine polonaise qui produit des jus de fruits et des petits pots pour bébé :

" "They can't tell us we don't know what we are doing, that we have nothing to contribute [en matière d'organisation du travail dans l'entreprise]. You can't say that all was bad under socialism. What was bad bad under socialism will be bad under capitalism, and it has to go. What was good under socialism will be good now, an dit has to stay." I aksed what was good and what had to stay. She replied, "well, everything. This was really a good firm. It's not like there wasn't an economy under socialism. We made profits, and that has to stay. There don't have to be many changes here, because this was the best firm in Poland." [...] She, like many other workers, believes that parts of the way work was organized under socialism are, in fact, suitable for use under the new constraints of the capitalist enterprise. Workers with long experience, she asserts, are not merely repositories of "bad habits" but have expertise that can make the firm successful as a capitalist enterprise ».

Elle montre ensuite comment les ouvriers sont à leur aise dans le travail en « flux tendu » promu par le nouveau management de l'entreprise, qui requiert de changer fréquemment de poste de travail et donc non seulement de maîtriser toutes les tâches mais aussi d'anticiper sur la prochaine activité afin de ne pas perdre de temps. Ce que le management présente comme une nouvelle organisation du travail, les ouvriers se l'approprient en réinterprétant leur expérience du socialisme, quand il fallait adapter la production non pas à la demande mais aux livraisons des coopératives, dont le contenu et la qualité étaient imprévisibles.

Selon Dunn, donc, la référence fréquente au passé socialiste ne doit pas être comprise comme une nostalgie ou un refus des changements, mais comme des ressources pour résister aux catégories projetées sur les ouvriers par les directions des entreprises persuadées de la rupture radicale entre le capitalisme flexible et "moderne" et le socialisme rigide, irrationnel et obsolète. Ce n'est pas "le socialisme » dans sa totalité qui est valorisé, ce sont certains éléments qui sont réutilisés pour se réapproprier son travail et valoriser son identité. Mais dans ce va et vient constant entre passé et présent les ouvriers courent le risque de rester identifiés au socialisme et donc au passé.

Au final, l'absence de visibilité statistique et d'attrait académique que présentent les ouvriers ne saurait surprendre. En effet si leur situation est dans l'ensemble sûrement pire que celles des autres salariés (en termes de risque de chômage en particulier), elle ne semble pas forcément leur être spécifique. L'intérêt heuristique de la séparation entre les ouvriers et les autres salariés d'exécution peut même à bon droit être questionné. Cependant ne faut-il pas inverser le raisonnement en suggérant que l'absence de catégories statistiques et de travaux de recherche sur cette catégorie la rend justement invisible? Le découpage en classes sociales, groupes sociaux ou catégories sociales est peut-être plus démodé dans les travaux de recherche et de statistique qu'inopérant dans la réalité sociale, mais à l'inverse, le travail de mise en forme du social par la statistique publique et par les sociologues n'est pas sans conséquences sur le statut des groupes sociaux et leur capacité à défendre leurs intérêts (Pierru et Spire 2008). Enfin ce travail a montré que les relations que les sociologues parviennent à établir avec les offices nationaux ou supranationaux de statistique sont essentielles car elles peuvent procurer aux sociologues des données riches sur des échantillons de bonne qualité (cas français), mais aussi au contraire, aboutir à produire à grands frais des informations sans pertinence pour la sociologie (cas tchèque et dans une certaine mesure 
OSC - Notes \& Documents $N^{\circ} 2009-02$

Marie Plessz - Les ouvriers en Europe centrale : la dissolutuion d'une catégorie sociale dans les statistiques

d'Eurostat). Ainsi la position institutionnelle de la sociologie conditionne étroitement sa capacité à produire un discours pertinent à la fois pour ses récepteurs et pour sa propre culture professionnelle.

\section{BIBLIOGRAPHIE}

Bafoil, François. 2000. "La classe ouvrière post-communiste. Des "héros au pouvoir" à l'exclusion des "petites gens" ». Genèses 39:74-97.

— (dir.). 2007. La Pologne. Paris: Fayard.

Beaud, Stéphane et Michel Pialoux. 1999. Retour sur la condition ouvrière : enquête aux usines Peugeot de Sochaux-Montbéliard. Paris: Fayard.

Blau, Peter M. et Otis D. Duncan. 1967. The American Occupational Structure. New York: Wiley.

Boltanski, Luc. 1982. Les cadres : la formation d'un groupe social. Paris: Éditions de Minuit.

—. 2008. Rendre la réalité inacceptable : à propos de "La production de l'idéologie dominante ". Paris: Démopolis.

Burawoy, Michael. 1988. "Peindre le socialisme ». Actes de la recherche en sciences sociales 75:75-82.

-. 2002. "What Happened to the Working Class? Errors of an Unrepentant Marxist ». Research in Social Stratification and Mobility 19 « The Future of Market Transition »:69-76.

Burawoy, Michael et János Lukács. 1992. The Radiant Past: Ideology and Reality in Hungary's Road to Capitalism. Chicago: University of Chicago Press.

Burawoy, Michael et Katherine Verdery (dir.). 1999. Uncertain Transition: Ethnographies of Change in the Postsocialist World. Lanham: Rowman \& Littlefield.

Chauvel, Louis. 2001. « Le retour des classes sociales ? ». Revue de l'OFCE:314-359.

Chenu, Alain. 1990. L'archipel des employés. Paris: INSEE.

Crowley, Stephen. 1997. Hot Coal, Cold steel : Russian and Ukrainian Workers from the End of the Soviet Union to the Post-Communist Transformations. Ann Arbor: University of Michigan Press.

Crowley, Stephen et David Ost. 2001. Workers after Workers' States: Labor and Politics in Postcommunist Eastern Europe. Lanham: Rowman \& Littlefield Publishers.

Desrosières, Alain et Laurent Thévenot. 1988. Les catégories socio-professionnelles. Paris: La Découverte.

Dunn, Elizabeth. 1999. « Slick Salesmen and Simple People: Négotiated Capitalism in a Privatized Polish Firm », p. 125-150 in Michael Burawoy et Katharine Verdery. Uncertain Transition, Ethnographies of Change in the Postsocialist World. Lanham: Rowman and Littlefield.

Erikson, Robert, John H. Goldthorpe et Lucienne Portocarero. 1979. «Intergenerational Class Mobility in Three Western European Societies ». British Journal of Sociology 30:415-441.

Gati, Charles et Jan F. Triska (dir.). 1981. Blue-Collar Workers in Eastern Europe. Londres: Allen \& Unwin.

Kolankiewicz, George. 1981. "Poland, 1980: the Working Class under "'Anomic Socialism"», p. 136-156 in Charles Gati et Jan F. Triska. Blue-Collar Workers in Eastern Europe. Londres: Allen \& Unwin.

Konopásek, Zdeněk (dir.). 2000. Our Lives As Database: Doing a Sociology of Ourselves, Czech Social Transitions in Autobiographical Research Dialogues. Prague: Charles University Press.

Kramer, Mark. 1995. "Polish Workers and the Post-Communist Transition, 1989-1993 ». Communist and Post-Communist Studies 28:71-114. 
OSC - Notes \& Documents $N^{\circ} 2009-02$

Marie Plessz - Les ouvriers en Europe centrale : la dissolutuion d'une catégorie sociale dans les statistiques

Kreidl, Martin. 2005. «Educating Communist Cadres: School Re-Entry and Sponsored Educational Mobility in State Socialism ». CCPR Working Paper Series: California Center for Population Research.

Monchatre, Sylvie. 2004. "De l'ouvrier à l'opérateur : chronique d'une conversion ». Revue française de sociologie 45:69-102.

Možný, Ivo. 1991. Proč tak snadno: některé rodinné důvody sametové revoluce : sociologický esej [Pourquoi si facilement? Quelques raisons familiales de la Révolution de Velours, essai sociologique]. Prague: Slon.

Parkin, Frank. 1969. «Class Stratification in Socialist Societies ». British Journal of Sociology 20:355-374.

Pierru, Emmanuel et Alexis Spire. 2008. «Le crépuscule des catégories socio-professionnelles ». Revue française de sociologie politique 58:457-481.

Portet, Stéphane. 2006. "Les paradoxes de la flexibilité du temps de travail en Pologne ». Toulouse: Université de Toulouse-Le Mirail.

Redor, Dominique. 1988. Les inégalités de salaires à l'Est et à l'Ouest. Paris: Economica.

Rowell, Jay. 2006. Le totalitarisme au concret : les politiques du logement en RDA. Paris: Economica.

Szalai, Erzsébet. 2006. "Ouvriers, relations de propriété et structure sociale ». La nouvelle alternative 21:171-183.

Sztompka, Piotr. 2002. "The Condition of Sociology in East-Central Europe », p. 548-558 in Max Kaase, Vera Sparschuch et Agnieszka Wenninger. Three Social Science Disciplines in Central and Eastern Europe: Handbook on Economics, Political Science and Sociology (1989-2001). Berlin: Lit Verlag.

Topalov, Christian. 1994. Naissance du chômeur, 1880-1910. Paris: Albin Michel. 